\section{$\underset{\substack{\text { hommes } \\ \text { \& migrations }}}{ }$}

Hommes \& migrations

Revue française de référence sur les dynamiques

migratoires

1302 | 2013

Le Japon, pays d'immigration?

\title{
Évolution du droit de la nationalité et individus métis : le cas des Nippo-Philippins
}

\section{Frédéric Roustan}

\section{(apenEdition}

\section{Journals}

\section{Édition électronique}

URL : http://journals.openedition.org/hommesmigrations/2466

DOI : 10.4000/hommesmigrations.2466

ISSN : 2262-3353

\section{Éditeur}

Musée national de l'histoire de l'immigration

\section{Édition imprimée}

Date de publication : 1 avril 2013

Pagination : 67-73

ISBN : 978-2-919040-22-3

ISSN : $1142-852 X$

\section{Référence électronique}

Frédéric Roustan, «Évolution du droit de la nationalité et individus métis : le cas des Nippo-Philippins », Hommes \& migrations [En ligne], 1302 | 2013, mis en ligne le 31 décembre 2015, consulté le 02 mai 2019. URL : http://journals.openedition.org/hommesmigrations/2466 ; DOI : 10.4000/ hommesmigrations.2466 


\title{
ÉVOLUTION DU DROIT DE LA NATIONALITÉ ET INDIVIDUS MÉTIS LE CAS DES NIPPO-PHILIPPINS
}

par FRÉDÉRIC ROUSTAN, chercheur invité en histoire sociale, université d'Hitotsubashi, Tokyo

\begin{abstract}
Fondée sur le droit du sang, l'acquisition de la nationalité japonaise n'est pas chose aisée pour les enfants nés de mère étrangère. Le cas des enfants philippo-nippons est révélateur à la fois des pesanteurs de la société nippone qui peine à donner une existence légale aux métis, et de sa dynamique d'ouverture. Depuis une trentaine d'années, les discriminations juridiques touchant les enfants nés d'unions mixtes ont tendance à disparaître grâce à la mobilisation d'organisations non gouvernementales composées de citoyens japonais et d'étrangers.
\end{abstract}

La place problématique des métis dans la société japonaise

Au cours des trente premières années de l'ère Meiji, le Japon adapte les concepts européens de hiérarchie des races, et l'influence de la pensée évolutionniste est reprise par de nombreux intellectuels japonais ${ }^{1}$. Ainsi, bien que l'histoire du métissage de populations au Japon ne débute pas au XIX ${ }^{\mathrm{e}}$ siècle ${ }^{2}$, c'est à cette époque que les interrogations soulevées par le darwinisme social font que les individus nés des relations intimes entre Japonais et étrangers, dont la présence augmente à partir du milieu des années 1850, commencent à être nommés ("konketsu" - métis, littéralement "sang mêlé"
- et "zasshu" - hybride), conceptualisés et étudiés ${ }^{3}$. Par ailleurs, la nécessité de se positionner dans la vision racialisante imposée par le colonialisme occidental accroît l'intérêt de l'élite japonaise pour le métissage des populations, notamment en tant qu'élément potentiel du processus de modernisation du Japon, pouvant contribuer à améliorer, ou à l'opposé mettre en péril, la "race Yamato". Peu de travaux nous permettent de comprendre dans le détail l'évolution socio-historique des populations métisses au Japon ${ }^{5}$. Le débat sur le métissage reste, jusqu'à 1945, quelque chose de marginal. Il 
semble cependant que la fluctuation des spéculations théoriques évolue loin de la réalité des interactions du quotidien. Assez rapidement, l'intérêt pour le métissage avec les Occidentaux devient minoritaire au regard de l'expansion japonaise en Asie ${ }^{6}$. La théorie de Davenport sur l'harmonie dans les métissages des "races proches" est une problématique influente mais le camp des prométissages est débordé pendant la guerre d’Asie Pacifique, la crainte d'une dissolution dans l'immensité continentale chinoise l'emportant ${ }^{7}$. En dehors des enfants de l'élite ${ }^{8}$, la majorité des premiers métis sont des enfants de prostituées qui constituent une population marginale dans les villes ouvertes aux étrangers ${ }^{9}$. Quant aux générations suivantes d'enfants métis, beaucoup sont victimes de discriminations ${ }^{10}$.

Entre 1945 et 1952, une nouvelle génération d'une dizaine de milliers d'enfants métis voit le jour du fait de la présence des forces militaires d'occupation, affectant profondément l'imaginaire de la société japonaise ${ }^{11}$. Dans le contexte de construction d'un mythe de l'homogénéité ethnique ${ }^{12}$, malgré certaines actions gouvernementales ${ }^{13}$, cette population métisse née de la défaite du Japon subit des discriminations d'autant plus fortes que sa visibilité expose ses origines aux yeux de tous ${ }^{14}$. Mais la situation évolue, conjointement à la situation migratoire du pays. Avant les années 1980, Okinawa, avec ses 30000 métis nippo-américains, fruit de l'occupation puis des bases américaines, focalise l'attention.

Entre les années 1980 et 1990, la presse commence à distinguer les origines ethniques en utilisant de nouvelles catégorisations qui rendent visibles pour la première fois des métis avec une parenté asiatique, traduisant la naissance de générations non plus seulement issues de la présence militaire américaine, mais des flux migratoires continus et croissants qui touchent le Japon depuis la fin des années $1970^{15}$. Actuellement, environ un enfant sur trente qui naît au Japon ${ }^{16}$ est considéré comme appartenant à cette catégorie de métis, soit, depuis le milieu des années 1990, plus de 20000 naissances par $a n^{17}$. Bien que les phénomènes d'assignation d'identité persistent, une évolution de la société japonaise allant vers plus d'inclusion est observable depuis les années $1990^{18}$. L'image monoethnique a partiellement cédé la place à celle d'une société japonaise constituée d'un groupe largement majoritaire et de divers groupes minoritaires, avec en arrière-fond le développement de théories et de politiques publiques locales dites "multiculturelles". Par ailleurs, nous constatons un phénomène d'empowerment : le discours produit par les métis n'est plus seulement celui de la victimisation, des groupes mobilisés ${ }^{19}$ se sont réappropriés et ont revendiqué des catégories comme "haaful half' (moitié) ou encore AmerAsian (une trentaine de termes, quasiment tous péjoratifs existent pour désigner les métis en japonais).

Cependant, contrairement aux pratiques de hiérarchisation du quotidien et des "racialisations cachées ${ }^{20 "}$, et de manière comparable à la situation en France, officiellement, hors du binôme national/étranger, le Japon ne distingue pas légalement ou administrativement de catégories ethniques ou raciales ${ }^{21}$. Ainsi, on est japonais ou étranger avec

6. Tōru Sakano, op. cit., pp. 189-200. 7. Ibid. 8. Voir Kenneth L. Richard, Dejima-Nagasaki-Japan-the World, the Nostalgic Journeys of Sadakichi Hartmann and Tomisaburo Kuraba, Two Men from the International Settlement, Kyoto, Kokusai Nihon bunka sentaa, 2000, pp. 4-3. 9. Shimonaka Kunihiko, Hoshō naki shakai (Une société sans protection sociale), Tokyo, Heibonsha.1960, p. 93-94. 10. Hirano Imao, Kijin no tiitaimu (Le Thé des aristocrates), Tokyo, Libroport, 1982, pp. 28-38 ; Hirano Imao, Remi ha ikiteiru (Remi est vivant), Tokyo, Kōdansha, 1979 (1e éd., Tōto Shobo, 1959), pp. 10-13. 11. Voir Ikutarō Shimizu, Seiichi Miyahara, Shōzaburō Ueda (dir.), Kichi no ko: kono jijistu wo dou kangaetara yoi ka (Les Enfants des bases : comment bien considérer cette réalité), Tōkyō, Kōbunsha, 1953. 12. Voir Eiji Oguma, Tan'itsu minzoku shinwa no kigen : nihonjin no jigazō no keifu (Aux origines du mythe de l'homogénéité ethnique : une généalogie des auto-représentations japonaises), Tōkyō, Shinyōsha, 1995. 13. Robert A. Fish, “Mixed blood Japanese”, in Michael Weiner (dir.), Japan's Minorities, London, Routledge, 2010, p. 46. 14. Un des premiers témoignages d'une fille de père afro-américain : Keiko Kozeki, Nihonjin Keiko : aru konketsu shōjo no shuki (Keiko la Japonaise : les Mémoires d’une jeune fille métisse), Tokyo, Bunkahōsōshuppan, 1967. 15. Lawrence Taguchi, Sengo Nihon ni okeru "konketsuji", "haafu", "amerasian" no sonzai / kioku; tabunka shakai heno (Mémoire et existence des "amerasiens", "haafu", "konketsu", dans le Japon d'après-guerre, vers une société muticulturelle), Tōkyō gaikokugo daigaku, Sotsugyoronbun, 2011, pp. 77-78. 16. Tokyo Shinbum, 4 août 2008. 17. http://www.mhlw.go.jp/toukei/saikin/hw/jinkou/suiiog/brth8.html 18. Stephen Murphy Shigematsu, "The invisible man and other narratives of living in the borderlands of race and nation", in David Blake Willis (dir.), Transcultural Japan, London, Routledge, 2008, pp. $283-286$. 19. Voir AmerAsian School in Okinawa, The 10 $0^{\text {th }}$ Anniversary of the AmerAsian School in Okinawa, 1998-2008, Ginowan, AASO, 2008. 20. William Wetherall, “The racialization of Japan”, in David Blake Willis (dir.), art. cit., pp. 264-281. 21. William Wetherall, “Nationality in Japan”, in Lee Soo Im, Shigematsu Stephen Murphy (dir.), Japan Diversity Dilemas, Lincoln, Universe, 2006, pp. 11-46 
une certaine diversité de visas encadrant ce dernier statut. L'État japonais, par le biais de sa législation sur la nationalité, définit l'appartenance au national et assigne une identité légale sans tenir compte des distinctions ethniques, religieuses ou autres ${ }^{22}$. Néanmoins, dans le cas des enfants nés d'un parent étranger, l'appartenance au national a été, et reste, inégalitaire vis-à-vis des enfants nés de deux parents japonais. Dans cet article, nous rappellerons l'évolution des inégalités juridiques affectant la nationalité des métis et nous analyserons la situation contemporaine avec un intérêt particulier pour les évolutions récentes de la loi permises grâce aux actions en justice menées par des enfants nés de couples nippo-philippins. Nous Base américaine de Yokosuka. (c) Camille Millerand utiliserons pour cela les résultats d'une enquête réalisée entre 2010 et 2012 au Japon et aux Philippines dans le cadre d'un programme post-doctoral JSPS (Japan Society for the Promotion of Science), au cours duquel nous avons notamment suivi les actions d'ONG investies dans le soutien aux Nippo-Philippins tentant d'obtenir la nationalité japonaise $e^{23}$.

\section{La nationalité des métis au Japon}

La législation régulant la nationalité au Japon est fondée sur le droit du sang, mais la transmission de la nationalité fut, jusquà l'amendement de 2008, totalement dépendante de la structure du couple dans lequel naissait l'enfant ${ }^{24}$. En 1873, les mariages dits "internationaux" au Japon acquièrent une existence légale. Cette loi poursuit la mise en place d'un système patrilinéaire unifié à tout le pays et initié par la loi sur l'état civil de 1871. Si une femme étrangère se marie à un Japonais, elle deviendra japonaise tout comme ses enfants. Si

une femme japonaise se marie avec un étranger, elle deviendra étrangère et perdra la possibilité de transmettre sa nationalité à ses enfants ${ }^{25}$. En 1899, la loi sur la nationalité renforce la patrilinéarité et le droit du sang, mais avec la possibilité donnée aux mères non mariées de transmettre leur nationalité japonaise, quelle que soit la nationalité du père. Par ailleurs, la naturalisation est rendue possible. Même si, dans les faits, la loi sur la nationalité discrimine les enfants dits "métis", la discrimination juridique ne vient pas d'une distinction visà-vis de l'origine ethnique des parents, mais d'une inégalité entre hommes et femmes à l'égard du mariage. En 1950, une nouvelle loi sur la nationalité est décrétée. Les femmes japonaises ne perdent plus leur nationalité en épousant un étranger, mais elles ne peuvent toujours pas la transmettre. Beaucoup d'enfants nés des troupes d'occupation alliées furent le fruit d'unions non maritales. Une grande partie d'entre eux furent abandonnés, une autre élevés par leurs mères, ce qui leur conféra la nationalité japonaise dans les deux cas (effet du droit du 
sol pour les enfants dont les parents sont inconnus). La situation n'évolue que beaucoup plus tard, sous l'action de mobilisations féministes, suite à la signature par le Japon de la Convention sur l'élimination de toutes les formes de discriminations à l'égard des femmes, conduisant à l'amendement de la loi en 1984 : les femmes japonaises mariées à un étranger peuvent enfin transmettre leur nationalité, avec un effet rétroactif sur vingt ans, l'enfant devant choisir une de ses deux nationalités avant sa majorité japonaise et abandonner l'autre, la double nationalité n'étant pas officiellement permise ${ }^{26}$. C'est d'ailleurs cette question de la double nationalité qui fut la raison fondamentale de la non-évolution de la loi : du fait des relations diplomatiques tendues avec les pays environnants, les politiciens japonais voulaient à tout prix éviter des situations de binationalité ${ }^{27}$.

Cependant, l'évolution de la loi en 1984 (appliquée en 1985) ne résout pas tous les problèmes que doivent affronter les enfants métis pour obtenir la nationalité japonaise et, jusqu'en 2008, la situation maritale des parents reste un facteur discriminant essentiel, touchant les enfants de mères étrangères, en particulier les enfants de mères philippines.

\section{Situation des Nippo-Philippins et nationalité japonaise}

Depuis les années 2000, les femmes philippines représentent environs $10 \%$ de la population étrangère vivant au Japon et sont la deuxième population d'étrangers mariés à des nationaux japonais ${ }^{28}$. De ces unions, maritales ou non, environ 77000 enfants sont nés au Japon depuis 1995, soit environ
300000 personnes depuis les années $1980^{29}$. Le phénomène migratoire à l'origine de la naissance de la population nippo-philippine du Japon débute vers la fin des années 1970. À cette époque, les contacts entre les Philippines et le Japon se multiplient, du fait de l'influence économique nipponne en Asie $^{30}$. Par ailleurs, à partir de 1973, l'administration Marcos $^{31}$ fait du tourisme l'un des principaux piliers du développement économique du pays, attirant notamment les hommes japonais intéressés par le tourisme sexuel ${ }^{32}$. Cependant, rapidement, insécurité et organisations féministes limitent le phénomène ${ }^{33}$ qui, néanmoins, ne disparaît pas mais évolue vers une nouvelle forme. Le flux de population est inversé et devient un mouvement migratoire des femmes philippines vers le Japon, destiné à pourvoir en main-d'œuvre le monde de la nuit qui commençait à en manquer ${ }^{34}$, alors même que la clientèle masculine gagnait en pouvoir d'achat ${ }^{35}$.

Cette migration est rendue possible par le gouvernement japonais qui accorde à ces femmes des visas de six mois dans la catégorie "entertainer 36 ", à la condition qu'elles aient suivi une formation artistique spécifique aux Philippines. Cependant, avant le durcissement de la loi en 2005 suite à des actions d'associations locales ${ }^{37}$ et des pressions internationales relatives aux tentatives de limiter le trafic d'êtres humains au Japon ${ }^{38}$, cette clause ne sera pas respectée. Ainsi, le nombre de ces visas accordés aux femmes philippines est très élevé et atteint son maximum en 2004, avec presque 90000 visas cette année-là. Rapidement, les médias enferment ces femmes "entertainer" dans la catégorie de "japayuki" (littéralement "aller au Japon") ${ }^{39}$. Ce terme, popularisé au milieu des années 1980, devient vite

25. Elle peut néanmoins «adopter son mari» qui devient alors japonais, et les enfants aussi. Au Japon, un mari peut être adopté par sa bellefamille. Cette pratique est généralement utilisée lorsqu'il n'y a pas de descendant masculin dans une famille, afin de conserver le nom et pouvoir perpétuer le culte des ancêtres. 26. Kiyoshi Hosokawa, “Japanese nationality in international perspective”, art. cit., pp. 188-19o. 27. Ibid., pp. 245-250. 28. Masaaki Satake, Mary Angeline Da-Anoy, Firipin-nihon kokusaikekkon, ijū to tabunkakyōsei (Les Mariages internationaux nippo-philipins, immigration et multiculturalisme), Tōkyō, Mekon, 2006. 29. Marilyn Erpelo (dir.), A Sneak Peek at Batis-YOGHI Development: A handbook on the Learning Experiences of Japanese-Filipino Children, Manila, Batis Center for Women, 20o9, p. 7. 30. Nobue Suzuki, “Filipino migrations to Japan : from surrogate Americans to feminized workers", in Shinji Yamashita (dir.), Transnational Migration in East Asia, Suita, National Museum of Ethnology, 2008, pp. 67-77. 31. Président des Philippines de 1965 à 1986. 32. Lieba Faier, Intimate Encounters, Filipina Women and the Remaking of Rural Japan, Berkeley, University of California Press, 2009, pp. 50-54. 33. Nobue Suzuki, "Between two shores, transnational projects and Filipina wives in/from Japan", in David Blake Willis (dir.), Transcultural Japan, op. cit., 2008, p. 67. 34. Si les Philippines sont les plus nombreuses, d'autres nationalités sont touchées par le même phénomène, comme les Thaïlandaises. 35. Masaaki Satake, “Filipina-Japanese intermarriages: A pathway to new gender and cross-cultural relations", in Asian and Pacific Migration Journal, $\mathrm{n}^{\circ}$ 4, vol. 13, 2004, p. 453.36. Rhacel Parreñas, Illicit Flirtations, Labor, Migration, and Sex Trafficking in Tokyo, Stanford, Stanford University Press, 2011, pp. 32-35. 37. Voir dans ce numéro l'article d'Hélène Lebail. 38. Ibid., pp. 32-35. 39. Ce mot s'inspire du mouvement prostitutionnel des femmes japonaises à l'étranger 
synonyme de l'équation $:$ femme asiatique $=$ objet sexuel ${ }^{40}$, avec les Philippines comme représentantes principales. Or, si pendant les premières années de ce phénomène migratoire les femmes philippines exercèrent effectivement cette activité, elles occupent dès la moitié des années 1980 des emplois n'incluant pas l'obligation de service sexuel ${ }^{41}$. Bien qu'une grande partie de ces femmes exercent d'autres types d'activités, leur image au Japon, grandement influencée par les stéréotypes marginalisant construits par les médias depuis vingt ans, n’a quasiment pas évolué ${ }^{42}$.

Favorisées en partie par leurs activités professionnelles, les relations entre ces jeunes femmes célibataires et les hommes japonais deviennent souvent intimes, avec parfois comme conséquence la conception d'un enfant. Or, avant 2008, lorsqu'un enfant naissait d'une mère étrangère et d'un père japonais hors mariage, il pouvait avoir la nationalité japonaise à condition que le père l'ait reconnu pendant la grossesse. Le père pouvait aussi reconnaitre l'enfant après la naissance mais il devait dans ce cas épouser la mère ${ }^{43}$.

Cette discrimination des enfants métis nés hors mariage n'est pas spécifique aux Nippo-Philippins, mais les cas sont particulièrement nombreux chez les premières générations d'enfants de mère philippine, en partie à cause du contexte des rencontres souvent liées au lieu de travail de ces femmes. Nombreux sont les pères déjà mariés lors de leur relation avec leur compagne philippine qui refusent de reconnaître l'enfant. Ces métis naissent donc étrangers bien que de père japonais. Dans le pire des cas, si la mère est en dépassement de visa, elle est expulsée aux Philippines avec son enfant et, si elle ne déclare pas l'enfant pour éviter l'expulsion, l'enfant devient alors apatride ${ }^{44}$. De plus, du fait de la durée limitée des visas, nombreuses sont les femmes qui tombent enceintes au Japon d'un homme japonais mais qui sont obligées de retourner aux Philippines, où l'enfant naît avec la nationalité de sa mère. Toutes les tentatives pour faire reconnaître ces enfants comme Japonais passent alors par une longue lutte juridique, avec l'aide nécessaire de divers acteurs mobilisés de la société civile.

\section{Mobilisations et amendement de la loi}

Dans les années 1980, les mobilisations se sont cristallisées autour des femmes philippines et de leurs problèmes au Japon : trafic d'êtres humains, violences domestiques, exploitations diverses, ou tout simplement difficultés pour obtenir un visa. Il faut attendre le début des années 1990, pour que la question des enfants soit mise en avant. Le sujet est abordé pour la première fois dans les médias en 1988, dans un programme de la télévision japonaise ${ }^{45}$. Une série d'émissions fait alors découvrir au grand public japonais l'existence de ces enfants. Toutefois, les situations présentées ne sont pas celles d'enfants vivant au Japon, mais aux Philippines. En 1991, un des Ces métis naissent étrangers bien que de père japonais. Dans le pire des cas, si la mère est en dépassement de visa, elle est expulsée aux Philippines avec son enfant et, si elle ne déclare pas l'enfant pour éviter l'expulsion, l'enfant journalistes ayant participé à devientalors apatride. ces reportages publie le premier ouvrage consacré au sujet ${ }^{46}$, fruit d'enquêtes conduites entre 1987 et 1988. À partir de cette publication, la presse japonaise aborde régulièrement le sujet, tout comme le reste des médias.

Depuis le début des années 1990, diverses associations japonaises et philippines sont actives dans le soutien aux mères philippines et à leurs enfants de 
père japonais. Nous présenterons ici seulement celle qui est à l'origine de l'évolution de la loi sur la nationalité.

En 1993, une association d'avocats bénévoles est fondée, le JFC-Bengodan, JFC signifiant "Japanese Filipino Children". Cette catégorie est créée pour remplacer le terme de "konketsuji" ("métis") jugé péjoratif et trop marqué historiquement. L'année suivante est créée, en complément du JFC-Bengodan, une autre association ${ }^{47}$ destinée à assister les avocats dans les relations avec leurs clients : contact et sélection des cas, recherche d'informations, traduction. En 1998, la structure est finalement complétée par une succursale à Manille (Maligaya House). Actuellement, ces trois structures sont regroupées sous le nom de JFC Network ${ }^{48}$. La principale activité de cette ONG est de rechercher le père des enfants. Ensuite, tout le travail consiste à lui faire reconnaître l'enfant et à le faire inscrire dans le registre familial et, si possible, à obtenir un soutien financier. L'inscription au registre familial permet d'obtenir un visa de résidence au Japon (catégorie "épouse ou enfant d'un Japonais") et de commencer une procédure juridique pour l'obtention de la nationalité (dite "naturalisation simplifiée"). Outre la question revendiquée par les ONG et les plaignants du "droit à la nationalité", la nationalité est aussi un enjeu important pour des raisons plus concrètes : elle représente l'espoir de pouvoir retourner ou rester au Japon, celui d'améliorer les conditions de vie des mères, tout comme celui d'offrir un meilleur avenir à leurs enfants.

La procédure juridique qui a conduit à l'évolution de la loi sur la nationalité commence le 12 avril 2005, lorsque neuf plaignants soutenus par le JFC Network décident d'attaquer l'État japonais. La raison qui les pousse à agir est un échec du processus de naturalisation, alors même qu'ils avaient obtenu la reconnaissance de filiation par leur père après la

Emma, 51 ans, et Jon-Michael, 24 ans, vivent paisiblement à Oppama, une ville proche de la base américaine de Yokosuka. Lui est originaire de l'Oregon. Quand sa mission à Tokyo se terminera, Emma ne sait pas encore si elle le suivra. (C) Camille Millerand

naissance. Le jour suivant est rendu le jugement d'un autre procès, pour un cas individuel identique : la loi de la nationalité est jugée anticonstitutionnelle par la cour régionale de Tokyo ${ }^{49}$. Presque un an plus tard, en mars 2006, le même jugement est rendu pour les neuf autres plaignants ${ }^{50}$. Cependant, ces deux décisions sont annulées par la Haute Cour de justice de Tokyo en février de l'année suivante. Les plaignants font appel et portent leur cas à la Cour suprême. Le dernier mot de cette épopée juridique est prononcé en juin 2008 : le premier jugement de non-conformité à la Constitution de la loi sur la nationalité est confirmé et la nationalité japonaise attribuée aux plaignants ${ }^{51}$. Suite à cela, le processus de révision de la loi est engagé pour aboutir au retrait de l'article 3-1 en décembre de la même anné $e^{52}$. Depuis, un enfant né hors mariage au Japon peut obtenir la nationalité si le père japonais le reconnaît, y compris après la naissance. 
Cependant, pour les enfants (de parents mariés ou non) qui naissent à l'étranger, reste en vigueur une limite dans le temps pour déclarer l'enfant et pour qu'il obtienne la nationalité japonaise. Dans leur cas, c'est l'article 12 de la loi sur la nationalité qui est en cause. Ainsi, si l'enfant possède automatiquement une autre nationalité à la naissance, que ce soit par le droit du sol ou par le droit du sang, les parents ont trois mois pour le déclarer aux représentants consulaires, après quoi il perdra automatiquement son droit à la nationalité japonaise. La majorité des enfants de parents nippo-philippins nés aux Philippines perdent leur nationalité japonaise dans le cadre de ces restrictions. Fort de sa victoire en 2008, le JFC Network défend, entre décembre 2010 et mars 2012, 27 cas d'enfants nés aux Philippines et ayant perdu leur nationalité japonaise du fait de l'article 12. À l'issue du procès, tous se voient refuser la nationalité japonaise, sauf celui d'entre eux qui résidait au Japon.

Parmi ces plaignants, certains avaient des parents mariés vivant en famille aux Philippines, mais ignorant la loi au moment de la naissance de leur premier enfant. Dans ces familles, généralement, les autres enfants possèdent la nationalité japonaise. Quelles que soient les raisons du retard de déclaration de la naissance (non-connaissance de la loi, empêchement, manque de moyens ou autres - la seule représentation consulaire se trouve à Manille), ces cas sont très nombreux aux Philippines. Cette forme de droit du sol discrimine les enfants métis nés à létranger car elle n'a pas lieu d'être au Japon, ni dans le cas de parents tous deux Japonais donnant naissance à l'étranger dans un pays de droit du sang.

La raison principale de cet échec est que, contrairement au procès qui a abouti au changement de la loi, dans son rendu de jugement, la Cour n'a pas considéré l'article 12 comme anticonstitutionnel. Par ailleurs, le juge en charge de l'affaire, Makoto Jozuka, a justifié le verdict en partie par léloignement physique et culturel des plaignants d'avec le Japon, ajoutant que cela les empêcherait de remplir leurs devoirs de nationaux japonais ${ }^{53}$. Les justifications apportées au verdict mettent en avant les éléments de droit du sol présents dans la définition de la nationalité japonaise. En outre, le verdict associe la nationalité à une culture, ce qui tend à exclure tout personne de double appartenance culturelle. Par ailleurs, il apparaît que cette culture ne pourrait pas s'acquérir puisque, bien qu'une majorité des plaignants aient entre 4 et 10 $a_{n} s^{54}$, léloignement est un argument décisif.

Malgré cet échec, les avocats du JFC Network ont porté le cas à la Haute Cour de justice de Tokyo en espérant que la décision précédente soit modifiée.

Le processus de lutte entre l'État et la société civile pour tenter de réformer la loi sur la nationalité continue. Des distinctions légales d'accès à la nationalité entre métis et non-métis subsistent, ainsi que de nombreux autres problèmes touchant les familles dites "mixtes" au Japon et leurs enfants, comme le rappelait encore il y a peu dans la presse un spécialiste du droit japonais ${ }^{55}$. Cependant, après les amendements de la loi sur la nationalité de 1985 et 2008, les principales discriminations juridiques touchant ces individus ont disparu, et une étape historique a été franchie dans leur intégration légale. Ces évolutions ont été rendues possibles par le militantisme de citoyens japonais et étrangers regroupés autour d'ONG et, en particulier, par l'engagement d'avocats qui, rappelons-le, ont depuis longtemps été un groupe d'acteurs particulièrement actifs dans les nombreuses luttes contre diverses formes de discriminations au Japon.

L'accès à la nationalité japonaise étant devenu naturel pour les enfants issus de couples mixtes, dans un proche avenir nous pouvons supposer que les mobilisations des populations métisses au Japon s'orienteront vers la reconnaissance de la double nationalité, officiellement illégale, mais qui pourtant existe dans les faits. 